\title{
Cambios en el proceso de enseñanza aprendizaje: desafíos en la práctica docente desde análisis de carrera universitaria chilena
}

Guzmán Sanhueza, Daniela; Castillo Leyton, Ana

Cambios en el proceso de enseñanza aprendizaje: desafíos en la práctica docente desde análisis de carrera universitaria chilena

Revista Educación, vol. 46, núm. 1, 2022

Universidad de Costa Rica, Costa Rica

Disponible en: https://www.redalyc.org/articulo.oa?id=44068165027

DOI: https://doi.org/10.15517/revedu.v46i1.45593

\section{(c) $(1) \Theta$}

Esta obra está bajo una Licencia Creative Commons Atribución-NoComercial-SinDerivar 3.0 Internacional. 


\title{
Cambios en el proceso de enseñanza aprendizaje: desafíos en la práctica docente desde análisis de carrera universitaria chilena
}

\author{
Changes in the Teaching-Learning Process: Teaching Practice Challenges based on Analysis of the University Major \\ in Chile
}

Daniela Guzmán Sanhueza

Universidad de Atacama, Copiapó, Chile, Chile

daniela.guzman@uda.cl

iD https://orcid.org/0000-0001-7905-309X

Ana Castillo Leyton

Universidad de Atacama, Chile

ana.castillo@uda.cl

iD https://orcid.org/0000-0003-0231-7135
DOI: https://doi.org/10.15517/revedu.v46i1.45593

Redalyc: https://www.redalyc.org/articulo.oa? $\mathrm{id}=44068165027$

Recepción: 17 Febrero 2021

Aprobación: 28 Marzo 2021

\section{Resumen:}

El propósito de este artículo es presentar los nuevos desafíos de la práctica docente derivados del análisis del perfil de quienes son protagonistas en el proceso de enseñanza-aprendizaje: estudiantes y docentes. Para ello se utilizó una metodología mixta, con técnicas de observación documental focalizada en la revisión de información secundaria disponible y encuesta al cuerpo académico de la unidad a través de la autoaplicación de un cuestionario online. Se evidencia en estudiantes la disminución de habilidades comunicativas y el aumento de actividad laboral paralela. En docentes se perfila una práctica flexible, comunicativa y que incentiva el trabajo colaborativo. Así, la práctica docente, bajo el modelo constructivista, se modifica en la medida que se presentan nuevas condiciones y escenarios de aprendizaje.

Palabras clave: Enseñanza y formación, Proceso de enseñanza y aprendizaje, Práctica docente, Docencia universitaria, Modelo educativo constructivista, Constructivismo, Perfil de ingreso estudiante.

\section{Abstract:}

This study proposes new challenges that arise during the teaching practice stage and stems from analysis of students and teachers, the actual protagonists of the teaching-learning process. This study is based on a mixed methodology using documentary observation techniques that focus on review of available secondary source information and a survey of the relevant faculty through an online survey. Results reveal a reduction in communication skills and an increase in parallel work activities. A more flexible, communicative practice has been emerging among teachers to encourages collaborative work. Teaching practices that adhere to a constructivist model, have been modified as we confront new conditions and learning scenarios.

KEYWORDs: Teacher Training, Teaching and Learning Process, Teaching Practice, University Teacher, Constructivist Educational Model, Constructivism, Student Entry Profile.

\section{INTRODUCCIÓN}

Desde su génesis, la Universidad representa un espacio privilegiado para la producción, distribución e intercambio de conocimientos; ha sido un lugar en donde se ha gestionado socialmente el saber y en este trayecto ha impulsado, en algunos casos, y ha debido asumir, en otros, los cambios sociales, políticos, económicos y culturales (Schwartzman, 2008). A través de una adecuada gestión del conocimiento, las facultades y departamentos de las instituciones de educación superior tienen amplias oportunidades de desarrollo a nivel de investigaciones interdisciplinarias; además de obtener una reducción de costos, nuevos servicios para sus estudiantes, estilos de enseñanza, asesorías y personalización (Correa-Díaz et al., 2019). 
Esta misión universitaria es compartida por toda la comunidad educativa y administrativa de la institución; sin embargo, quien implementa los procesos iniciales que apuntan a la generación y gestión del conocimiento son los cuerpos académicos, que deben adaptarse y responder a un escenario pedagógico en transición, a través de una práctica docente que responda a la diversidad estudiantil.

Existe un perfil de estudiantes que ingresan a la carrera de trabajo social de la Universidad de Atacama que presenta ciertas tendencias, necesarias de evidenciar, para conocer los requerimientos en la formación y ajustar el proceso de enseñanza aprendizaje.

Este desafío, pretendido a los cuerpos académicos de la universidad, será definido, entre otros elementos, por las características del estudiantado que ingresa a la carrera de trabajo social de los años 2016 al 2018 y el perfil docente de la esta. Se analizan ambos para responder positivamente al requerimiento de un modelo constructivista que privilegia el aprendizaje situado, experiencial, comprometido y crítico.

\section{ANTECEDENTES O ESTADo DE LA CUESTIÓN}

La incorporación de elementos teóricos que expliquen el aprendizaje como un proceso de construcción ha permitido instalar un nuevo paradigma en educación, cuya base teórica radica en la teoría del conocimiento constructivista de Vygotsky (1978). Se resalta establecer el carácter colectivo del conocimiento, la relevancia del proceso y la construcción de este. Se ha generado un cambio de paradigma de aprendizaje en donde se valora la interacción social, de manera que se establece que el estudiantado aprende más eficazmente cuando lo hace de forma cooperativa. (Tigse, 2019). En este sentido, la concepción de la otredad como sujeto que contiene habilidades, capacidades y potencialidades que le permiten, de manera autónoma, tomar decisiones y ejercer derechos, ha permeado los campos de la educación superior.

El primer cambio fundamental, reflejo de este cambio de paradigma, responde a centrar la educación en el aprendizaje y no en la enseñanza. Hasta hace un tiempo, la idea de definir con precisión y focalizar el éxito de los aprendizajes en los contenidos era aceptada y asumida por la mayoría del profesorado. Ahora, el foco está en entregar a la población estudiantil estrategias dirigidas a la resolución de problemas de la profesión, por medio del desarrollo de competencias del ser, hacer y convivir (Alonzo et al., 2015).

Otro elemento relevante es entender el aprendizaje como un proceso y no como un producto, lo que implica dejar de concebir que los contenidos o resultados del aprendizaje sean lo que se modifica con el propio aprendizaje, la que sería el objeto o producto de este. Es decir, desde esta lógica, el aprendizaje es acumulativo o transformador. En cambio, si se valora el aprendizaje como un proceso, se hace en referencia a los mecanismos cognitivos y la actividad mental empleada por la persona que está aprendiendo. Es decir, este último corresponde a lo que las personas aprendices hacen o pueden hacer para cambiar sus conocimientos. Finalmente se reemplaza enseñar contenidos por enseñar a aprender. El respaldo pragmático de esto lo da la misma definición de conocimiento en la sociedad actual que lo caracteriza con base en su creciente caducidad, pues, aunque existe conciencia de los conocimientos necesarios para la actualidad, no hay certeza de la vigencia de estos. Ciertamente el estudiantado debe obtener el conocimiento como producto, pero debe entender las condiciones en que este se aplica. Solo así podrá determinar cuándo es necesario poner ese conocimiento en duda y gestionar nuevos conocimientos con criterios propios para decidir la validez de algún constructo teórico o práctico para una situación dada, tal como menciona Taborda (2005):

La enseñanza, como se ha dicho, no se opone ni niega al aprendizaje, más bien lo garantiza. Pero ocurre que este aprendizaje se relaciona más con competencias para la vida, con la posibilidad de acceder a la crítica, a la solución de problemas, a la construcción de estrategias cognitivas para comprender la ciencia y las disciplinas que, con el establecimiento de una base de memoria inflexible en el individuo (p.3).

En coherencia con lo expuesto, Calderón (2020) plantea que "los procesos de aprendizaje y enseñanza en contexto de formación profesional se caracterizan por situar al estudiante en una modalidad de 
aprendizaje sustancialmente diferente a la lógica tradicional." (p. 207), pone en reflexión el protagonismo en las actividades de aprendizaje que se tienen en el aula, proceso basado en la capacidad meta cognitiva del alumnado, al procurar un aprendizaje colaborativo, significativo y autónomo. Estos deben estar intencionados por un currículum adecuado y personal docente capacitado para gestionar estrategias que faciliten su desarrollo. El actual modelo de aprendizaje, la adherencia al enfoque por competencias e instalación de procesos de aseguramiento de la calidad en la educación superior, han incentivado profundos cambios en la Universidad, los que conllevan alteraciones en las funciones, roles y tareas docentes asignadas; al mismo tiempo exigen un mayor desarrollo de nuevas competencias para ejercer de manera amplia y adecuada sus funciones.

La educación superior, a nivel global, exhibe un nuevo perfil de estudiantes, el cual demanda importantes desafíos para las instituciones. Si bien en Chile todavía el acceso a la educación superior sigue estando altamente correlacionado con el nivel de ingresos (Ministerio de Desarrollo Social de Chile, 2015), en la actualidad existe un aumento constante y paulatino de estudiantes que ingresan a la educación superior. Sin embargo, se mantienen 15 puntos porcentuales menos que la media de los países pertenecientes a la Organización para la Cooperación y el Desarrollo Económicos [OCDE] (Organización para la Cooperación y el Desarrollo Económicos, 2018).

Este panorama podría llevar a pensar que el aumento relativo del ingreso a la educación superior, asociado a la gratuidad, implica mayores posibilidades de lograr equidad en la educación. La experiencia muestra que las desigualdades de base impactan en la permanencia, titulación oportuna, progreso y desempeño en la actividad académica (Quintena, 2013).

\section{REFERENTES CONCEPTUALES Y TEÓRICOS}

El Modelo educativo de la Universidad de Atacama, centrado en la teoría constructivista del aprendizaje, manifiesta, desde su incorporación, diversas complejidades en la implementación y adaptación a las carreras. Si bien, Vygotsky enfatiza la influencia de los contextos sociales y culturales en la apropiación del conocimiento y pone gran énfasis en el rol activo del estudiantado, también establece la importancia de considerar un rol docente activo en el proceso, es la persona facilitadora del desarrollo de estructuras mentales en el estudiantado, para que este sea capaz de construir aprendizajes más complejos (Ledesma-Ayora, 2014).

En respuesta a este desafío, ha surgido la discusión sobre las características, competencias o habilidades que debe demostrar el personal docente en el ejercicio de su quehacer, el cual demanda conocimiento en herramientas metodológicas, didácticas y evaluativas pertinentes a las características de este nuevo paradigma (Walker, 2016). Diferentes estudios han concluido que la enseñanza de una disciplina específica se sustenta en el dominio de un conjunto de conocimientos (pedagógicos, curriculares, del estudiantado, del contexto y del contenido que se enseña, entre otros), en consecuencia, para enseñar una temática específica no basta el conocimiento disciplinar (Tovar y Cárdenas, 2010).

Una investigación realizada a doce personas académicas de las mejores universidades de Estados Unidos revela interesantes resultados para explicar el éxito académico de docentes de nivel universitario. Bain (2007) muestra que el profesorado extraordinario, definido como aquel que ha conseguido "influir en la manera de pensar, actuar y sentir de sus estudiantes" (p.15), cuenta con sólidos y actualizados conocimientos en lo específico de su campo disciplinar, logra crear ambientes colaborativos de aprendizaje, captar la atención del grupo de estudiantes, establecer compromisos y generar la interacción con problemas reales, lo que permite el contraste de conocimientos y la realidad. Todo esto es sumado a la motivación que generan en el estudiantado, el incentivo en el manejo de su propio aprendizaje, la transmisión de una gran confianza para alcanzarlo y el compartir su propia experiencia de aprendizaje con el fin de generar la reflexión. En una investigación realizada en México en 2013, se establece que una docencia exitosa es aquella en donde, junto con el reconocimiento de su carácter multifuncional, se desarrolla una estrecha relación profesorado- 
alumnado, una metodología de evaluación clara y equitativa, y un método de enseñanza participativo. Desde los resultados se proyecta que el personal docente bien evaluado en una de éstas características, lo será también en las demás (Rocha, 2013).

Se requiere una persona docente bien preparada para dar continuidad y consistencia a los sistemas educativos. La transmisión de conocimientos a través de modelos de explicación homogéneos dentro del aula y en el horario previsto, podrá dedicarlos ahora a la guía de forma individual y diferenciada de su alumnado y grupos asignados (García, 2012).

Sin embargo, es muy común encontrar docentes que son profesionales, que conocen su disciplina, pero que no necesariamente aprendieron cómo enseñarla (Tovar, 2011). Se establece, por otro lado, que quienes se desempeñan como docentes de educación superior tienden a repetir la forma de enseñanza que recibieron (la mayoría en un marco de desarrollo positivista de la docencia universitaria) y, por tanto, se visualizan resistencias a nuevas formas de establecer el proceso enseñanza-aprendizaje. (Monereo, 2010).

La universidad ha pasado largo tiempo sin preocuparse por lo que se denomina pedagogía universitaria. La mayor parte de las personas académicas no cuenta con formación didáctica. Más bien, se ha ido generando paulatinamente y en respuesta a los procesos de aseguramiento de la calidad, dispositivos orientados a esta tarea. Como lo menciona Mas (2011), el personal docente universitario trabaja en la institución formativa de mayor nivel existente y, curiosamente, su inmensa mayoría no se han formado para ejercer esa función, ya que han ingresado en este cuerpo docente después de formarse largamente en los contenidos propios de su área, sin recibir ningún tipo de formación pedagógica; mayoritariamente no han sentido esta necesidad formativa, ni la institución les ha exigido ninguna formación pedagógica previa al desarrollo de dicha función docente.

En la literatura pertinente, se indica que existen 3 dimensiones que interactúan entre sí en la actividad docente y que se desarrollan con igual importancia: personal, disciplinar y pedagógica (Francis, 2006). Según investigaciones anteriores, orientadas a determinar los elementos que se relacionan con el logro educacional pretendido, quienes que toman mayor protagonismo dentro de la dimensión personal son: la motivación hacia el estudiantado, comunicación efectiva, respeto hacia la población estudiantil, coherencia entre el discurso y la praxis (Rojas, 2011). Respecto a la dimensión disciplinar, lo planteado anteriormente indica que se refiere al conocimiento disciplinar de un área específica de la disciplina, el profesional ha desarrollado cierta experticia (Ortega, 2017). En la dimensión pedagógica, quienes desempeñan docencia de educación universitaria deben manejar la didáctica general y la didáctica específica (Tovar y García, 2014). La primera, derivada de la teoría del aprendizaje constructivista, corresponde al manejo de las estrategias metodológicas que aseguren el eficaz desarrollo del proceso de aprendizaje; la segunda se orienta a la especificidad epistemológica disciplinar. Por tanto, se refiere al rol docente como agente, que, a través de los procesos de enseñanza, orienta, dirige, facilita y guía la interacción de sus estudiantes con el saber colectivo culturalmente organizado (Guzmán et al., 2015).

No existe una didáctica universitaria, sino hábitos entre docentes que permiten cierta organización del proceso. Esto se refleja en que existe una incoherencia entre lo que se declara y la práctica docente. En la línea expuesta, se proponen 4 niveles de formación de quienes forman, establecidos según el momento de desarrollo profesional, estos se especifican a continuación:

\footnotetext{
una formación previa, dirigida básicamente a personal con posibilidades de iniciar la carrera universitaria (por ejemplo, becarios de investigación); formación inicial, ofertada a los profesores noveles, normalmente con escasa experiencia docente y con una notoria precariedad laboral; formación continua, dirigida a profesores con experiencia y adaptada a las necesidades concretas del propio docente, de su universidad, departamento, etc.; formación especializada en enseñanza disciplinar, orientada a aquel profesor universitario con una larga trayectoria profesional, que por su propia inquietud y por la necesidad de la institución donde labora, desee dedicarse al diseño y aplicación de planes de estudios, de innovación, de mejora de la calidad, etc. (Mas, 2011, p.16).
}

Por otro lado, existe todo un sistema estructurado tradicional de funciones asociadas a la docencia universitaria, clasificadas en las áreas de: docencia, cargada de desafíos en torno a un nuevo modelo de 
enseñanza-aprendizaje en el que se entiende que es un proceso continuo bidireccional; investigación, que nutre la docencia de nuevos conocimientos, los actualiza; y gestión, que puede participar activamente en la gestión de su organización y que es fundamental para desarrollar las dos anteriores (Sanz et al., 2014). Existe una valorización diferenciada de las dos funciones principales del profesorado universitario: investigación y docencia. El desajuste que existe entre ellas y el predominio de la investigación sobre la docencia, motiva que el avance hacia nuevas concepciones de la enseñanza y el aprendizaje complejo (Caballero y Bolívar, 2015). La investigación ha primado por sobre la docencia, tiene como refuerzo a ello un sistema de incentivos monetarios, jerárquicos, reconocimiento entre pares y de los/as estudiantes. La producción investigadora tiene una clara primacía sobre la calidad docente como lo menciona Lucarelli (2008), existiendo una relación directa entre investigación y poder. El poder que ya no se ejerce en las aulas, producto de una democratización del proceso de enseñanza-aprendizaje, se concentra en la actividad investigadora, pues suele pensarse que quien investiga entregará una mejor docencia, más actualizada de la disciplina. Ya hace 17 años atrás, Morán (2004), establece la necesidad de repensar la vinculación de la docencia y la investigación como una modalidad asumida en las universidades para profesionalizar la docencia y revisar las reales posibilidades de impactar en la vida académica.

\section{Metodología}

La investigación tiene por objetivo analizar los cambios en la práctica docente, vinculados al perfil del estudiantado de la Carrera de Trabajo Social de la Universidad de Atacama, Chile. La hipótesis que se deduce de este objetivo es: existen características del perfil de estudiantado que repercuten en la práctica docente, generando nuevos desafíos.

Se configura como un estudio de caso, situado en la carrera de trabajo social de la Universidad de Atacama, Chile, de carácter público y regional. Es de tipo mixta, pues incorpora la revisión de fuentes secundarias, específicamente documentos hipertextuales correspondientes a fichas de estudiantes aplicadas al ingreso a la carrera y estadística interna del Departamento de Trabajo Social. Esta información se adiciona a la obtenida por la aplicación de cuestionario online al cuerpo académico de la unidad, quienes son la población de esta investigación, con el propósito analizar ambos perfiles y así proyectar los desafíos para la puesta en práctica de un modelo de aprendizaje constructivista. En la Tabla 1 se resumen las técnicas utilizadas y se especifica el objeto de análisis en cada una de ellas.

TABLA 1

Técnicas utilizadas en metodología mixta y sujetos de investigación.

\begin{tabular}{l|l}
\hline TÉCNICAS UTILIZADAS & OBJETO DE ANÁLISIS \\
\hline $\begin{array}{l}\text { Observación documental y análisis } \\
\text { hipertextual de informes institucionales. }\end{array}$ & $\begin{array}{l}\text { Características de entrada de } 180 \text { estudiantes correspondientes a } \\
\text { ingreso de } 2016 \text { a } 2018 \text { de la carrera de Trabajo Social. }\end{array}$ \\
\hline Cuestionario autoaplicado online. & $\begin{array}{l}\text { Opinión de } 8 \text { docentes de la carrera de Trabajo Social, } \\
\text { correspondientes al 100\% del cuerpo académico vigente a 2020. }\end{array}$ \\
\hline
\end{tabular}

Fuente: elaboración propia, 2020.

En la Tabla 1 se establece la utilización de la observación documental, la cual se realiza a través de pauta de observación y cuestionario, el cual incorpora la operacionalización de las tres dimensiones de la práctica docente (Francis, 2006; Rojas, 2011; Ortega, 2017), que permiten perfilar las tendencias actuales de esta en la carrera. El instrumento es autoaplicado, anónimo y contiene preguntas mixtas, en donde cada docente podrá seleccionar todas las opciones que le represente. Dentro de las preguntas se han considerado preguntas control, orientadas a comprobar la veracidad de las respuestas (Hernández et al., 2014). 
La Tabla 2 presenta las dimensiones consideradas en la práctica docente para esta investigación., orientadas a lo personal, disciplinar y pedagógico.

TABLA 2

Dimensiones de la práctica docente.

\begin{tabular}{|c|c|c|}
\hline DIMENSIÓN & SUBDIMENSIÓN & CATEGORÍA \\
\hline \multirow{5}{*}{$\begin{array}{l}\text { Dimensión personal: referida a aquellas } \\
\text { habilidades blandas desarrolladas como } \\
\text { competencias genéricas o transversales. }\end{array}$} & \multirow[t]{2}{*}{ Motivación } & Tipo de motivación (interna o externa) \\
\hline & & Mecanismo para motivar al estudiantado \\
\hline & \multirow{2}{*}{$\begin{array}{l}\text { Comunicación } \\
\text { efectiva }\end{array}$} & Jerarquía en la comunicación \\
\hline & & Lenguaje utilizado \\
\hline & Coherencia & $\begin{array}{l}\text { Encadenamiento y complementariedad de lo que se dice } \\
\text { y lo que se hace. }\end{array}$ \\
\hline \multirow{6}{*}{$\begin{array}{l}\text { Dimensión disciplinar: orientada a configurar el } \\
\text { comportamiento docente en el desarrollo del } \\
\text { proceso de enseñanza de los aspectos } \\
\text { teóricos-metodológicos, éticos-políticos y } \\
\text { técnico-operativos del trabajo social. }\end{array}$} & Formación & $\begin{array}{l}\text { Coherencia de la formación obtenida con la formación } \\
\text { entregada. (Per fil propio versus perfil al que tributa). }\end{array}$ \\
\hline & \multirow[t]{2}{*}{ Experiencia } & Conocimiento de distintos espacios laborales \\
\hline & & Años de experiencia previa al ingreso a la academia \\
\hline & \multirow{3}{*}{$\begin{array}{l}\text { Actualización del } \\
\text { conocimiento } \\
\text { disciplinar }\end{array}$} & Flexibilidad y apertura a nuevos conocimientos \\
\hline & & Estrategias de actualización \\
\hline & & Educación continua \\
\hline \multirow{3}{*}{$\begin{array}{l}\text { Dimensión pedagógica: conjunto de actividades } \\
\text { intencionales y planeadas para facilitar que el } \\
\text { estudiantado se apropie y reconstruya alguna } \\
\text { porción del saber. }\end{array}$} & \multirow[t]{2}{*}{ Didáctica general } & Aplicación del modelo constructivista de aprendizaje. \\
\hline & & Implementación de competencias genéricas \\
\hline & $\begin{array}{l}\text { Didáctica específica } \\
\text { para el trabajo social }\end{array}$ & $\begin{array}{l}\text { Utilización de estrategias metodológicas para integrar } \\
\text { aprendizajes de cada asignatura a la disciplina. }\end{array}$ \\
\hline
\end{tabular}

Fuente: elaboración propia, 2020.

La elaboración del cuestionario incorpora los criterios de consistencia, lo que implica tratar de que con el menor número de preguntas se obtenga la mejor información y claridad; evitar preguntas ambiguas o que sugieran respuestas; discreción, y facilidad.

\section{Resultados}

\section{Perfil de entrada de estudiantes de Trabajo Social, Universidad de Atacama}

Según el informe anual de perfil de ingreso de la carrera de Trabajo Social, el estudiantado en su mayoría corresponde a mujeres, egresadas de establecimientos públicos y que mencionan la vocación como factor de ingreso y elección de la carrera. Además, cerca del $20 \%$ de quienes ingresan menciona tener experiencia previa con el servicio a través del voluntariado o acción social en sus comunidades, lo que refuerza el fundamento vocacional.

En la Figura 1 se indica que entre los años 2016 y 2018 ha aumentado la cantidad de estudiantes que trabaja, pues ha pasado de un 15,69\% en 2016 a un 31,43\% en 2018, tal como se evidencia a continuación: 


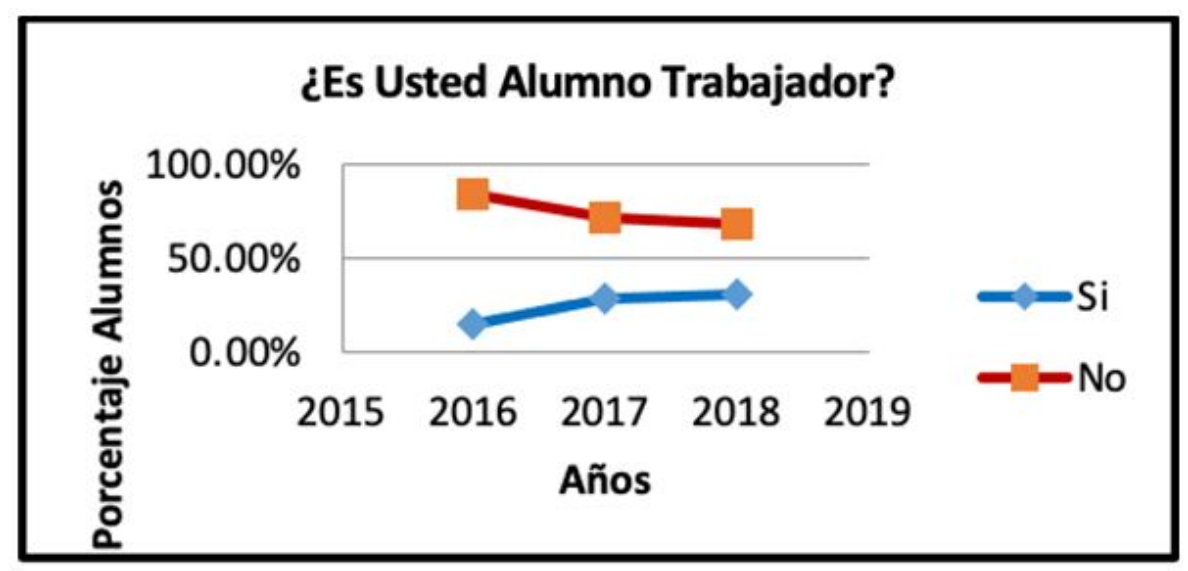

FIGURA 1.

Estudiantes que trabajan.

Fuente: elaboración propia en base a informe CTA.

Se proyecta que existen diferencias en la disposición de tiempos para asumir el trabajo autónomo, donde este es uno de los componentes principales del modelo pedagógico constructivista. El doble o triple rol desarrollado en la actualidad por el estudiantado (padre, madre, estudiante y persona que trabaja) refleja el estado de tensión y presión constante que debe sobrellevar. Especialmente si se considera que la mayoría de quienes ingresan a la carrera son mujeres, la desigualdad existente en la ocupación del tiempo se explica por la brecha de género en la asunción de responsabilidades de cuidado. En términos generales, son mayoritariamente mujeres quienes tienen la responsabilidad de cuidados (hacia padres, madres, hijos e hijas). Respecto a la necesidad de compatibilizar los estudios con el trabajo, la mayoría de quienes se encuentra trabajando lo hace en tiempo parcial.

Otro elemento relevante en la motivación que el alumnado desarrolla en los primeros años de la carrera radica en la razón por las cual ingresó a esta y que desde la literatura se indica como un predictor de la permanencia y desempeño. En la Figura 2 se expone la razón manifestada por quienes ingresan a la carrera durante el periodo de 2016 a 2018.

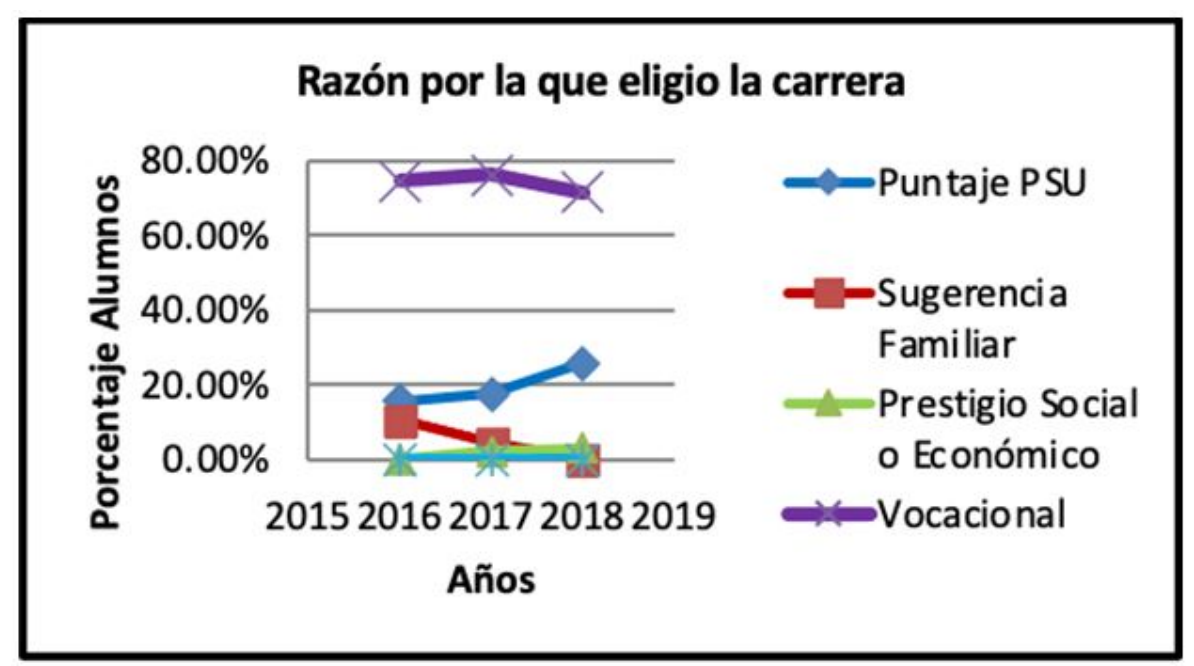

FIGURA 2.

Razón de elección de la carrera, estudiantes de Trabajo Social.

Fuente: elaboración propia en base a informe CTA. 
Como se observa, existe un porcentaje mayoritario de estudiantes que menciona el aspecto vocacional como aquel que motiva su ingreso a la carrera de Trabajo Social. Sin embargo, este porcentaje ha disminuido comparativamente entre los años 2017 y 2018. A diferencia, el puntaje obtenido en la Prueba de Selección Universitaria (PSU) ha mostrado, en promedio y durante el mismo periodo, un aumento significativo de un $15,78 \%$ en 2016 a un $25,7 \%$ el 2018.

Respecto a las habilidades comunicativas del alumnado que ingresa, la evaluación inicial indica un desarrollo menor de estas habilidades, tal como se muestra en la Figura 3:

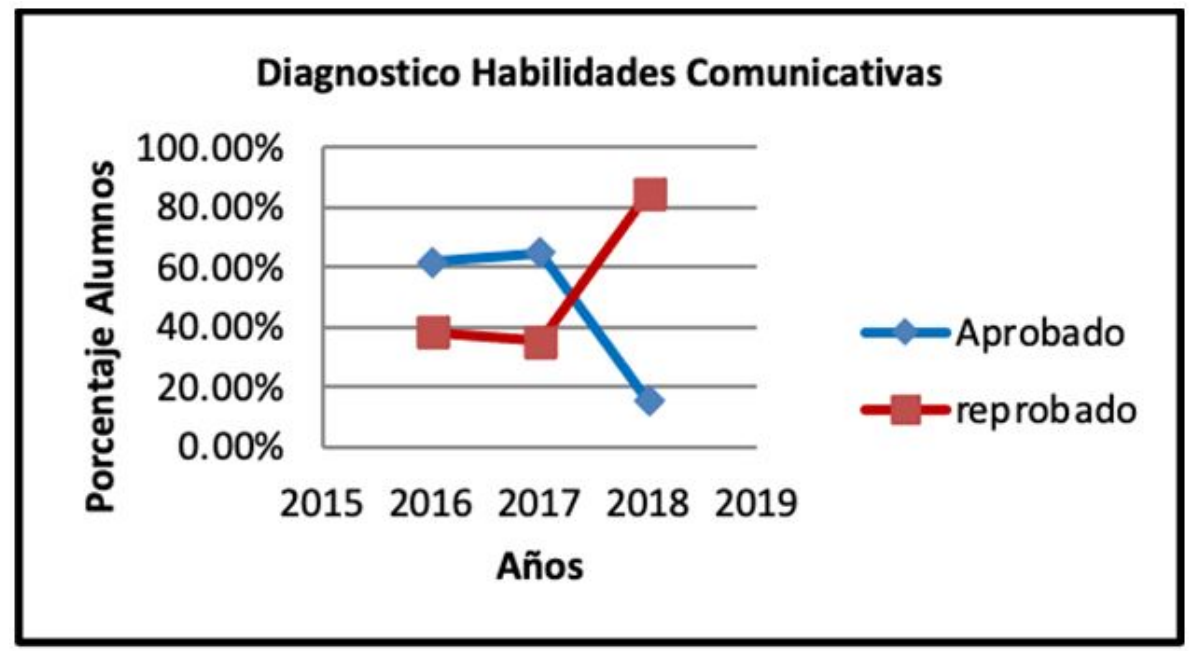

FIGURA 3.

Habilidades comunicativas.

Fuente: elaboración propia en base a informes CTA.

Una competencia relevante para la carrera de trabajo social, es la capacidad para desarrollar interacciones comunicativas efectivas. Sin embargo, desde la información obtenida, las habilidades comunicativas en el estudiantado han disminuido con el tiempo (Figura 3); esto hace cada vez más evidente el déficit y la necesidad de abordarlas. Se observa una drástica disminución en las habilidades comunicativas que presenta el alumnado de ingreso del año 2018 en relación con los años anteriores, lo cual representa una preocupación para el equipo docente, pues las metodologías en el aula consideran el aprendizaje colaborativo y colectivo, y, con ello, las habilidades comunicativas como un elemento basal para el buen desarrollo del proceso de enseñanza-aprendizaje.

\section{Perfil docente de la carrera de Trabajo Social, Universidad de Atacama}

El perfil docente corresponde a aquellas características con las que el cuerpo académico de la carrera de Trabajo Social cuenta y que le hacen distinguirse de otros cuerpos académicos. Se caracteriza por ser un equipo constituido por profesionales de distintas disciplinas afines al Trabajo Social, tales como Antropología, Psicología y Sociología. El grupo de personas académicas de la carrera de Trabajo Social de la Universidad de Atacama está compuesto mayoritariamente por mujeres profesionales, con grado académico y que se encuentran en un rango de edad entre los 27 y los 56 años. La experiencia profesional es considerada fundamental para la realización de la docencia universitaria, ya que, cada vez más, se requiere situar los aprendizajes a los requerimientos del medio. A continuación, en la Figura 4, se presenta en detalle los años de experiencia profesional del cuerpo académico de trabajo social. 


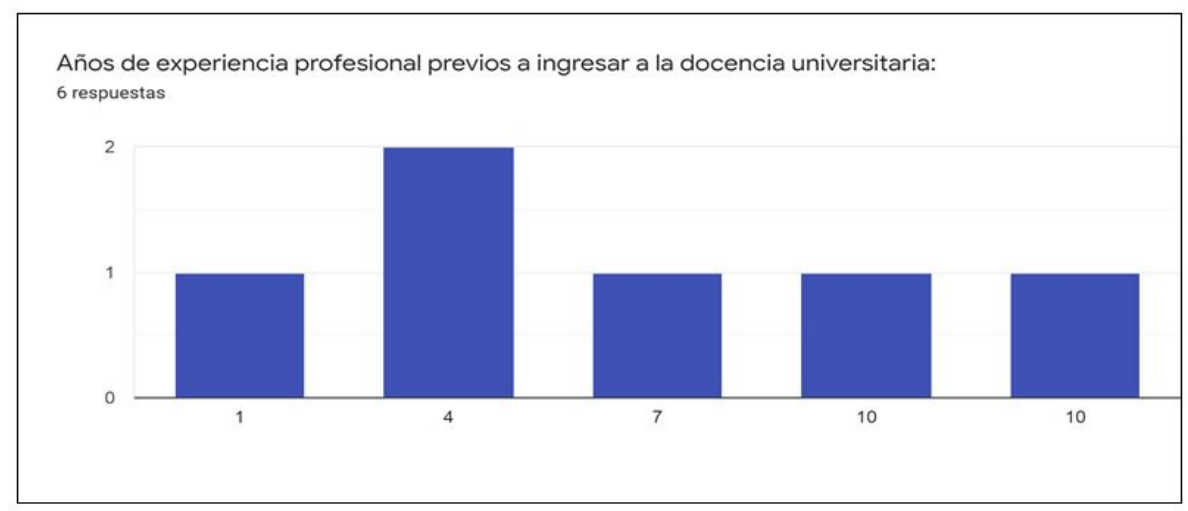

FIGURA 4.

Experiencia profesional previa a la docencia universitaria.

Fuente: elaboración propia.

Según la Figura 4, la mayoría cuenta con 4 años o más de experiencia profesional previa a la docencia, característica relevante en una profesión en donde la práctica profesional es fundamental para entender las adecuaciones disciplinarias en las distintas áreas de desempeño. Esta experiencia se focaliza principalmente en las áreas de salud, comunidad, infancia, migraciones, mujeres, juventudes y ruralidad.

Donoso (2001), dos décadas atrás, plantea que quien ejerza docencia universitaria tiene el desafío de cumplir un rol en el proceso de enseñanza-aprendizaje diferente al que cumplían sus docentes en la época en que se formó. Al consultar al equipo docente de la Universidad de Atacama al respecto, el 83 \% menciona que son conscientes de las diferencias entre la formación recibida en sus casas de estudio de origen y la que hoy implementan en el aula, tanto en los énfasis formativos (perfiles de egreso) como en las formas de desarrollar la docencia (didáctica). Sin embargo, y ante el reconocimiento de esta brecha, el equipo mantiene una actualización temática, disciplinar y pedagógica, en la utilizan mecanismos (Figura 5) que permiten mantener una retroalimentación permanente con el medio y con el estado del conocimiento.

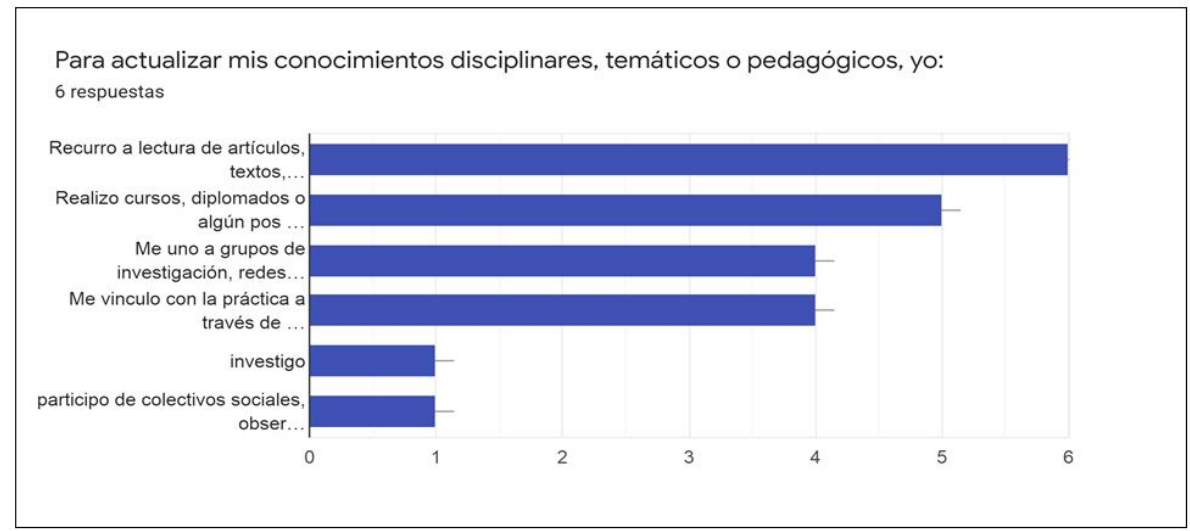

FIGURA 5.

Mecanismos de actualización del cuerpo docente.

Fuente: elaboración propia.

En la Figura 5 se distingue que una persona manifiesta utilizar la investigación como medio para actualizar conocimientos y cuatro mencionan vincularse a grupos o redes de investigación, lo cual evidencia la importancia de esta para nutrir la práctica docente.

La Figura 6 muestra las respuestas a la consulta sobre la forma de desarrollar la clase y aquellos elementos indispensables. El cuerpo académico valora mayoritariamente crear ambientes colaborativos de aprendizaje y conocer las características del estudiantado. Esto concuerda con las premisas establecidas por el modelo 
pedagógico constructivista y lo planteado por Vygotsky, quien da protagonismo en el aprendizaje a la interacción social, condicionada por el contexto y las características individuales (Tünnermann, 2011). Luego, a contar con material de apoyo, principalmente audiovisual; finalmente, dejar las reglas claras, preparar con anticipación la clase, ser fiel a esa planificación y realizar trabajos en grupo.

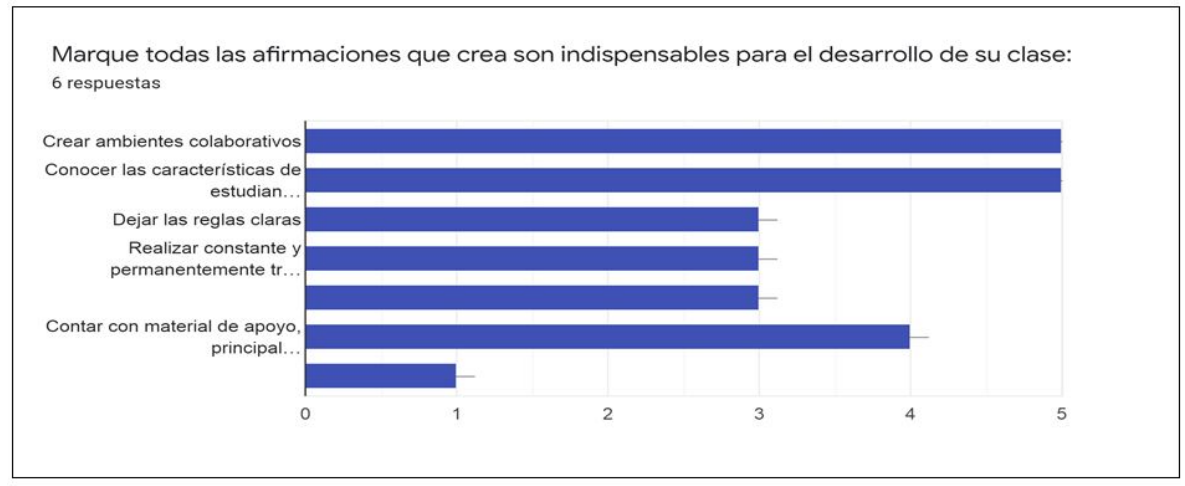

FIGURA 6.

Desarrollo de la clase.

Fuente: elaboración propia.

Complementario a esta información, se establece que, durante el desarrollo de la clase, las personas académicas se preocupan por motivar a sus estudiantes, para ello utilizan metodologías participativas y aplican en sus explicaciones elementos teóricos a la práctica. Las metodologías participativas, propias del nuevo paradigma de aprendizaje centrado en el estudiantado, es relevante en el nuevo perfil docente, ya que, a través de ellas, se hace factible cumplir con el rol de facilitar el aprendizaje. Se realiza una reconstrucción del contenido disciplinar, específicamente para promover el aprendizaje, lo que requiere un cuerpo docente con la capacidad de transformar el conocimiento de la disciplina en formas pedagógicamente útiles. Así también, se realiza la explicación del propósito formativo de la asignatura y su implicancia en el ejercicio profesional, específicamente al vincular los niveles de dominio comprometidos de las competencias disciplinares con el perfil de egreso. Esta acción, junto a la vinculación con el conocimiento previo y la aplicación de los contenidos a contextos próximos y cotidianos, son parte de las condiciones necesarias para proyectar un aprendizaje significativo en el estudiantado.

Existen elementos transversales a la práctica docente que influyen en la percepción de los ambientes de aprendizaje. Por ejemplo, el tipo de comunicación que se establece entre estudiante y docente es una expresión de la relación de alteridad que se da entre ambas partes y que se dispone a la puesta en práctica del proceso de aprendizaje. Las características de la comunicación entre estudiantes y docentes, según lo planteado por este último grupo, se presentan en la Figura 7 de la siguiente manera: 


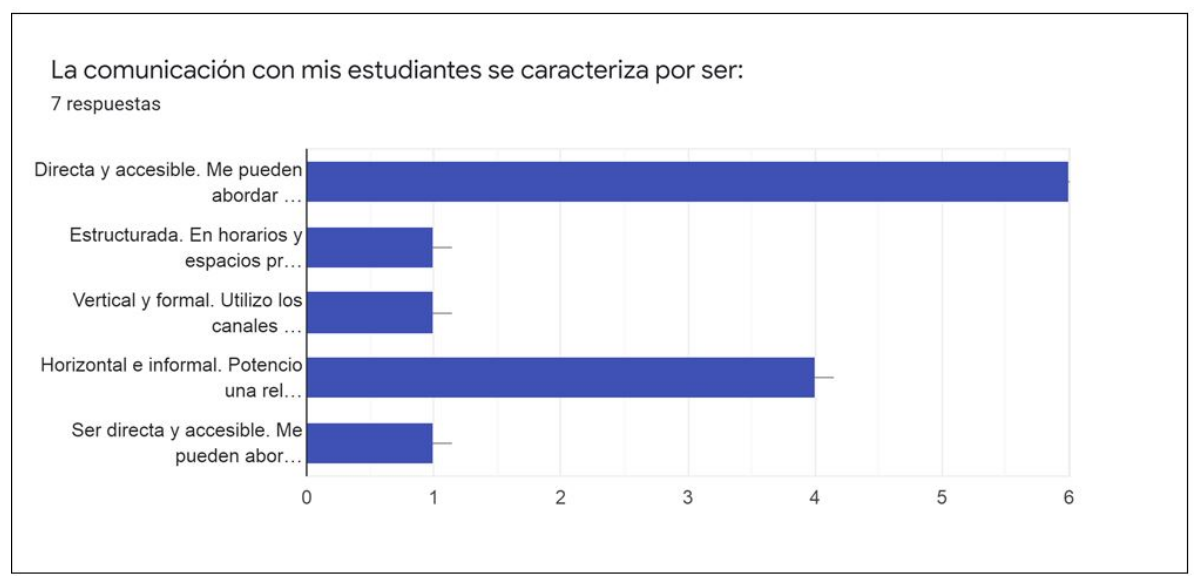

FIGURA 7.

Características de la comunicación estudiante-docente.

Fuente: elaboración propia.

La competencia comunicativa es considerada como parte de la dimensión personal del perfil docente, que incorpora las acciones asociadas al ámbito de empatía en las relaciones docente-estudiante. Destacan aspectos como la comunicación verbal y no verbal, y la manera en que desarrolla el proceso de enseñanza-aprendizaje. En la totalidad de las respuestas se menciona que el tipo de comunicación que se establece es directa y accesible, lo que permite resolver de forma oportuna los requerimientos del estudiantado. Complementario a esta información, en preguntas anteriores del cuestionario, se establecen prácticas en el aula durante el desarrollo de la clase, orientadas a la comunicación, que favorecen los espacios de intercambio de opiniones, establecimiento de acuerdos, retroalimentación directa, entre otras iniciativas.

De igual manera, el $100 \%$ de las respuestas indica que la forma de resolver conflictos se presenta como una oportunidad formativa, ya que, ante la existencia de una situación problemática, se refuerzan competencias transversales y genéricas, que pertenecen a las habilidades deseadas e indispensables en profesionales del siglo XXI, tales como el respeto, la tolerancia y el trabajo en equipo.

\section{Desafíos en la práctica docente en relación con el perfil estudiantil}

\section{Promover la autonomía y gestión del conocimiento}

El modelo del sistema escolar tradicional primario y secundario se caracteriza por ser directivo, pues existen elementos estructurales de este que potencian y perpetúan dicho modelo. Por ejemplo: la cantidad de estudiantes por curso, las exigencias curriculares basadas en transferencia de contenidos, sistema de medición de resultados del aprendizaje, sistema de subvención, etc. (Maldonado y Espinosa, 2018).

El estudiantado que ingresa a la universidad debe no solo asumir el protagonismo de su proceso de aprendizaje, sino también desaprender la forma instructiva que caracterizó sus años en la formación primaria y secundaria. De manera complementaria, el cuerpo docente, consciente de este déficit, utiliza distintas estrategias para reforzar habilidades. Por ejemplo, incentiva el trabajo autónomo y la autogestión a través de revisiones bibliográficas, elaboración de diseños de proyectos y de anteproyectos de investigación. En coherencia con el modelo educativo, esto se hace con la priorización del trabajo colectivo, colaborativo y el aprendizaje con otras personas. Cabe señalar que dentro del creditaje de cada asignatura se consideran horas presenciales y horas autónomas. 


\section{Desarrollar el protagonismo en su proceso de aprendizaje}

Considerar al estudiantado como el centro del proceso de aprendizaje representa la idea de que asumen la autoría y acción final de este; ya no solo se focaliza en la recepción del conocimiento, mirada por lo demás muy positivista, sino más bien se convierte en protagonista del proceso, donde demuestra voluntad para la búsqueda autónoma de conocimiento. El nuevo paradigma imperante presenta un estudiantado activo, dinámico, con aprendizaje previo que le permita anclar el nuevo, que demanda de sus docentes no solo el manejo teórico y conceptual, sino también el manejo pedagógico, didáctico y metodológico, con capacidad para auto motivarse y auto dirigir su proceso (Solórzano-Mendoza, 2017). Sin embargo, en las aulas, la mayoría de las veces se encuentran estudiantes que tienden a seguir directrices, con motivaciones diversas que no necesariamente se asocian al conocimiento. Se presentan situaciones contingentes que hacen complejo el conciliar la vida universitaria con las responsabilidades personales y cotidianas. La feminización de la carrera de trabajo social se expresa prematuramente en el ingreso mayoritario de estudiantes mujeres, muchas de las cuales cuentan con responsabilidades de cuidado o domésticas.

Así también, el estudiantado debe lidiar con deficiencias en su formación primaria y secundaria, que se refleja en dificultades para comprender lo que leen, ratificado por el bajo rendimiento en comprensión lectora en pruebas nacionales (DEMRE, 2020).

\section{Incentivar el pensamiento critico}

Dentro del perfil de egreso comprometido en la carrera de trabajo social se establece el pensamiento crítico como sello profesional. Son 4 competencias sello que deben desarrollarse durante el transcurso curricular completo correspondiente a 10 semestres. En este sentido, el cuerpo académico de la carrera debe considerar acciones, tanto en el aula como fuera de ella, que potencien el desarrollo de estas competencias en cada asignatura, al incorporar estos contenidos de manera transversal en la malla curricular. El desafío más relevante en este ámbito refiere al desarrollo de la argumentación, lo cual se relaciona directamente con la comprensión lectora y hábitos basales, como gusto por la lectura, que permiten contar con mayores recursos para respaldar opiniones y posturas. En este desafío se requiere promover estrategias asociadas a la expresión y debate de ideas, para fundamentar posturas y valorar el diálogo como medio para la resolución de problemas.

\section{Potenciar competencias transversales y genéricas}

De acuerdo con los requerimientos profesionales del siglo XXI, las competencias transversales y genéricas se han convertido en fundamentales a la hora de evaluar la calidad profesional. En el caso del trabajo social; la disposición al trabajo en equipo, la inclusión, liderazgos democráticos, respeto a la diversidad, búsqueda de la calidad, entre otras, son competencias que se desarrollan durante la carrera; esto adiciona un desafío extra a la labor docente.

Ya no basta con orientar el proceso de enseñanza-aprendizaje solo a competencias disciplinares o específicas. Es importante destinar tiempo dentro de cada asignatura para motivar el desarrollo de elementos actitudinales referidos a competencias transversales y genéricas, y evaluarlos, para con ello generar procesos de retroalimentación a través de instrumentos coevaluativos, autoevaluativos y heteroevaluativos. 


\section{Conclusiones}

Uno de los desafíos para la práctica de personas académicas actuales tiene relación con la función que hoy cumplen en el proceso de enseñanza aprendizaje. Los nuevos escenarios representan una forma distinta de entender el aprendizaje, responden a un paradigma distinto, que implica una relación diferente entre docente-estudiante, lo que implica un cambio en la dialéctica de la enseñanza aprendizaje en torno a la formación profesional. Por consiguiente, es fundamental reflexionar sobre los nuevos desafíos de la práctica docente y la necesaria definición de quienes son protagonistas en el proceso: estudiantes y docentes. Por ello se realizó revisión en el contexto de la carrera de Trabajo Social de la Universidad de Atacama, donde se evidencia la complejidad de la puesta en práctica de un modelo de aprendizaje constructivista, que requiere de disposiciones y conocimiento previo por parte de sus protagonistas; además, da cuenta de que la práctica docente, bajo este modelo, se modifica en la medida que se presentan nuevas condiciones y escenarios de aprendizaje.

Si se aborda el perfil de entrada del estudiantado a la carrera de Trabajo Social de la Universidad de Atacama, se evidencia que la mayoría ingresa por vocación; así se establece que esta razón intrínseca es la que define la decisión de elección de la carrera profesional. La definición vocacional considera las características propias y distintivas del grupo estudiantes, junto a proyecciones y habilidades; este es un proceso personal, analítico que se realiza en momentos previos al ingreso a la educación superior. Con este análisis a favor, se proyecta un mejor desempeño y mayor adherencia al proyecto formativo.

La carrera de trabajo social busca potenciar un estudiantado crítico, reflexivo y dinámico a través de un proceso activo de formación. Sin embargo, según perfil de ingreso, las características que se presentan distan de la autonomía y autorregulación necesaria para que este modelo de aprendizaje se instale de forma adecuada con todas sus potencialidades. En este contexto, la autonomía representa una competencia fundamental, que permite abordar el proceso y asegura el potencial cambio de conocimientos en cada sujeto. Tanto así que les corresponde a los cuerpos académicos facilitar el desarrollo de la autonomía del estudiantado en el proceso de aprendizaje; en consecuencia, debe disponer de los dispositivos metodológicos necesarios para el logro de esta. Este proceso es distante con lo potenciado en los años de escolaridad anterior, ya que la educación en Chile (básica y secundaria), cuyo modelo de formación es tradicional, estandarizado y no desarrolla aspectos estratégicos o reflexivos del propio aprendizaje del estudiantado, potencia un proceso de aprendizaje dirigido, que busca que el estudiantado siga instrucciones y modelar el proceso de aprendizaje de manera generalizada. Es así como en las aulas se debe primero romper ese esquema y luego fomentar la autonomía, desde ahí hay un transitar que complejiza la enseñanza y el aprendizaje.

Así también, la situación de doble y triple rol que trae cada estudiante dispersa las energías, dificulta el desarrollo de la autonomía y condiciona la participación en la vida universitaria, tal como lo mencionan los estudios previos: en las aulas se encuentra, la mayoría de las veces, estudiantes que tienden a seguir directrices, con motivaciones diversas que no necesariamente se asocian al conocimiento, con situaciones contingentes que le hacen complejo el conciliar la vida universitaria con las responsabilidades personales y cotidianas.

En la misma línea, las debilidades en las habilidades comunicativas del estudiantado, evidenciadas en el brusco descenso de estas en las cohortes de ingreso, junto a la necesidad de conciliar vida familiar, laboral y académica, representan las características relevadas por los informes de perfil de ingreso del estudiantado. Dichos resultados indican una configuración compleja para la implementación del modelo de aprendizaje que requiere de un estudiantado activo, autónomo y crítico. De manera complementaria, se evidencia un perfil docente en coherencia a las tres dimensiones: disciplinaria, personal y pedagógica; en cada una de ellas se dispone el valor del estudiantado como sujeto activo y protagonista del proceso de aprendizaje. Se logra evidenciar una relación basada en la comunicación directa, horizontal y respetuosa, que generan acciones motivadoras para el alumnado, tanto en el aula como en la actitud docente fuera de ella, y la coherencia del discurso y la acción. Respecto a la dimensión disciplinar, la formación del cuerpo académico, en distintas 
casas de estudios y con diferentes especializaciones, se pone a disposición de un proyecto formativo de trabajo social, derivado de un perfil de egreso co-construido. La formación de posgrado del cuerpo académico, magister y doctorado, indica generación de conocimiento específico que reportan a las áreas de formación y las líneas de investigación de la carrera de Trabajo Social y corresponden al mecanismo formal de perfeccionamiento académico. Respecto a las prácticas de actualización del conocimiento disciplinar, se valora la investigación y la vinculación a grupos o redes de investigación, lo cual permite instalar la disciplina en el ámbito de las ciencias sociales de manera articulada y no como acciones aisladas.

En la dimensión pedagógica, el cuerpo docente de la carrera de Trabajo Social, maneja principalmente la didáctica general, ya que la totalidad cuenta con diplomado en Docencia Universitaria, que contiene todos los elementos conceptuales y metodológicos derivados de la teoría del aprendizaje constructivista y que son aplicados a la práctica docente a través de la utilización de estrategias participativas que potencien el aprendizaje significativo, colaborativo y colectivo. Esto se complementa con la forma horizontal de comunicación que caracteriza la relación docente-estudiante, basada en el respeto del rol que cada parte cumple.

\section{Recomendaciones}

En síntesis, y de acuerdo con el perfil del estudiantado que ingresa a la carrera, los desafíos que deben asumir las personas docentes son aún más provocadores que lo mencionado por la literatura, ya que, bajo el modelo constructivista, quien se desempeña como docente adquiere un rol de activador de disposiciones necesarias para que el estudiantado asuma el protagonismo en su proceso de aprendizaje. Todo esto, además, debe considerar la diversidad de formas de aprendizaje del estudiantado que ingresa. Se necesita instalar una práctica docente flexible y atenta a los constantes requerimientos nacientes, motivados tanto por el perfil del estudiantado como por las exigencias del medio laboral (perfil de egreso). Para ello se proyecta retomar un trabajo conjunto entre docentes por nivel, a través de actividades académicas integradas entre asignaturas que contribuyen a la misma competencia.

Finalmente, el perfil del estudiantado tiene características específicas que no han sido consideradas para la puesta en práctica de un nuevo modelo educativo, que requiere, por parte de este grupo, mayores destrezas o, en su defecto, disposiciones específicas para desarrollar estas destrezas y habilidades. Al no ser completamente desarrolladas en los años de escolaridad anterior, deben asumirse como parte de la práctica docente en las carreras universitarias, consideradas en los procesos de renovación curricular o, de manera más inmediata, para establecer acciones remediales. Algunas universidades utilizan cursos de nivelación, bachilleratos y otras actividades complementarias al currículo, para favorecer este proceso.

\section{REFERENCIAS BibLIOGRÁFICAS}

Alonzo, D., Vargas, J., Valencia, M. y Bolívar, N. (2015). Estrategias para el desarrollo de competencias en el aula, con enfoque socio formativo. Boletin Redipe, 4(9), 77-85. https://dialnet.unirioja.es/descarga/articulo/6232397.pdf

Bain, K. (2007). Lo que hacen los mejores profesores universitarios. (2da. edición). Ediciones Universidad de Valencia.

Caballero, K. y Bolívar, A. (2015). El profesorado universitario como docente: hacia una identidad profesional que integre docencia e investigación. Revista de docencia Universitaria, 13(1), 57-77. http://red-u.net/redu/files/jo urnals/1/articles/900/public/900-3924-1-PB.pdf

Calderón, M. (2020). Desafíos para el aprendizaje en las prácticas progresivas en la formación de profesores. Una aproximación cualitativa en una universidad chilena. Profesorado, Revista de Currículum y Formación de Profesorado, 24(2), 202-222. https://doi.org/10.30827/profesorado.v24i2.14075

Correa-Díaz, A., Benjumea-Arias, M y Valencia-Arias, A. (2019). La gestión del conocimiento: Una alternativa para la solución de problemas educacionales. Revista educare, 23(2), 1-27. http://dx.doi.org/10.15359/ree.23-2.1 
DEMRE. (2020). Informe del cálculo de puntajes PSU admisión 2020. [Informe técnico ADM 2020]. https://demre. cl/estadisticas/documentos/informes/2020-calculo-puntaje-proceso-admision-2020.pdf

Donoso, S. (2001). Nuevo rol docente, nuevos desafíos de la docencia. Revista Calidad en la Educación, (15), 1-11. h ttp://dx.doi.org/10.31619/caledu.n15.445

Francis, S. (2006). Hacia una caracterización del docente universitario "excelente": Una revisión a los aportes de la investigación sobre el desempeño del docente universitario. Revista Educación, 30(1), 31-49. https://www.red alyc.org/pdf/440/44030103.pdf

García, L. (2012). Sociedad de conocimiento y educación. Editorial Aranzadi S. A. https://bit.ly/2NguAru

Guzmán, C., Campaner, G., y Gallino, M. (2015). Dimensión pedagógica - didáctica en docentes universitarios. El caso de Ingeniería. Revista Argentina de Enseñanza de la Ingeniería, 4(8), 9-18. https://www.researchgate.net/publi cation/278708405_Dimension_pedagogica_didactica_en_docentes_universitarios_El_caso_de_Ingenieria

Hernández, R., Fernández, C., Baptista, P. (2014). Metodología de investigación. Editorial McGraw-Hill.

Ledesma-Ayora, M. (2014). Análisis de la teoría de Vygotsky para la reconstrucción de la inteligencia social. Universidad Católica de Cuenca.

Lucarelli, E. (2008). Asesoría pedagógica y cambio en la Universidad. Profesorado, Revista de Currículum y Formación de Profesorado, 12(1), 1-14. http://www.ugr.es/ recfpro/rev121ART7.pdf

Maldonado, H. y Espinosa, M. (2018). Prospectiva y emancipación social: aprendizaje creador. Retos y propuestas en la transformación educativa. Editorial Amapsi. https://bit.ly/3jD3bfy

Mas, O. (2011). El profesor universitario, sus competencias y formación. Revista El profesorado, 15(3), 195-211. htt p://www.ugr.es/ recfpro/rev153COL1.pdf

Ministerio de Desarrollo Social, Chile. (2015). Informe de Desarrollo Social 2015. Ministerio de Desarrollo Social. ht tps://www.desarrollosocialyfamilia.gob.cl/pdf/upload/IDS_INAL_FCM_3.pdf

Monereo, C. (2010). ¡Saquen el libro de texto! Resistencia, obstáculos y alternativas en la formación de los docentes para el cambio educativo. Revista de Educación, 352, 583-597. http://www.revistaeducacion.educacion.es/re35 2/re352_26.pdf

Morán, P. (2004). La docencia como recreación y construcción del conocimiento sentido pedagógico de la investigación en el aula. Revista Perfiles educativos, 26(105-106), 41-72. http://www.scielo.org.mx/scielo.php?p $\mathrm{id}=$ S0185-26982004000100003\&script $=$ sci_abstract\&tlng=es

Organización para la Cooperación y Desarrollo Económicos [OCDE]. (2018). Education at a Glance 2018: OECD Indicators. OECD Publishing. P. http://dx.doi.org/10.1787/eag-2018-en

Ortega, J. (2017). Conocimiento escolar y conocimiento "disciplinar" del profesor: algunas reflexiones sobre la participación del profesor en la construcción y enseñanza del contenido asociado a las disciplinas escolares. Revista Folios, 45(1), 87-102. https://doi.org/10.17227/01234870.45folios87.102

Quintena, G. (2013). Desigualdades sociales y educación superior, el proceso de toma de decisiones de estudiantes desde un enfoque sociológico. En M. Irigoin, R. del Valle y M. Ayala (Eds.). Acceso y permanencia en la educación superior: sin apoyo no hay oportunidad (pp. 53-95). https://aequalis.cl/publicaciones/acceso-y-permanencia-enla-educacion-superior-sin-apoyo-no-hay-oportunidad/

Rocha, R. (2013). Escala de opinión de los estudiantes sobre la efectividad de la docencia (eoeed) en educación superior. Formación universitaria, 6(6), 13-22. https://dx.doi.org/10.4067/S0718-50062013000600003

Rojas, C. (2011). Ética profesional docente: un compromiso pedagógico humanístico. Revista Humanidades: Revista de la Escuela de Estudios Generales, 1(1), 1-22. https://dialnet.unirioja.es/descarga/articulo/4920530.pdf

Sanz-Blas, S., Ruiz-Mafé, C. y Pérez-Pérez, I. (2014). El Profesor universitario y su función docente. Revista Espacio I +D Innovación Más Desarrollo, 3(5), 97-112. https://doi.org/10.31644/imasd.5.2014.a05

Schwartzman, S. (2008). Universidad y desarrollo en Latinoamérica: experiencias exitosas de centros de investigación. Editorial Instituto Internacional de la UNESCO para la Educación Superior en América Latina y el Caribe. h ttps://bit.ly/3psv7UM 
Solórzano-Mendoza, Y. (2017). Aprendizaje autónomo y competencias. Dominio de las Ciencias, 3, 241-253, https:/ /dominiodelasciencias.com/ojs/index.php/es/article/view/390

Taborda, C. (2005). Orientación universitaria: Marco conceptual de referencia. Repertorio De Medicina y Cirugía, 14(1), 1-4. https://doi.org/10.31260/RepertMedCir.v14.n1.2005.368

Tigse, C. (2019). El Constructivismo, según bases teóricas de César Coll. Revista Andina De Educación, 2(1), 25-28. https://doi.org/10.32719/26312816.2019.2.1.4

Tovar, J. (2011). La competencia docente universitaria: alternativa teórica. Papeles, 3(6), 43-50. http://186.28.225.7 $0 /$ index.php/papeles/article/view/279

Tovar, J. y Cárdenas, N. (2010, 22-26 febrero). Formación inicial de docentes en el marco de las actuales perspectivas en investigación educativa: la complejidad. [Ponencia]. IV Congreso de Transdisciplinariedad, Complejidad y Eco formación.

Tovar, J. y García, A. (2014, 3-4 julio). Características de la práctica docente universitaria: caminos hacia la formación permanente de los docentes. [Ponencia]. En M. Tortosa, J. Álvarez, N. Pellín (Eds.) XII Jornadas de Redes de Investigación en Docencia Universitaria. El reconocimiento docente: innovar e investigar con criterios de calidad. Alicante, España. http://hdl.handle.net/10045/40144

Tünnermann, C. (2011). El constructivismo y el aprendizaje de los estudiantes. Universidades. Revista Universidades, (48), 21-32. https://www.redalyc.org/pdf/373/37319199005.pdf

Vygotsky, L.S. (1978). Mind in Society: Development of Higher Psychological Processes. Harvard University Press.

Walker, V. (2016). El trabajo docente en la universidad: condiciones, dimensiones y tensiones. Perfiles educativos, 38(153), 105-119. http://www.scielo.org.mx/scielo.php?script=sci_arttext\&pid=S0185-2698201600030010 5

\section{INFORMACIÓN ADICIONAL}

Cómo citar: Guzmán-Sanhueza, D. y Castillo-Leyton, A. (2022). Cambios en el proceso de enseñanza aprendizaje: desafíos en la práctica docente desde análisis de carrera universitaria chilena. Revista Educación, 46(1). http://doi.org/10.15517/revedu.v46i1.45593 